\title{
RADIAL OSCILLATION OF HARMONIC FUNCTIONS IN THE KORENBLUM CLASS
}

\author{
YURII LYUBARSKII AND EUGENIA MALINNIKOVA
}

\begin{abstract}
We study radial behavior of harmonic functions in the unit disk belonging to the Korenblum class. We prove that functions which admit two-sided Korenblum estimate either oscillate or have slow growth along almost all radii.
\end{abstract}

\section{INTRODUCTION AND MAIN RESULTS}

We study radial behavior of functions harmonic in the unit disk $\mathbf{D}$. Let $\operatorname{Harm}(\mathbf{D})$ stay for the set of all real-valued functions harmonic in $\mathbf{D}$ and $|E|$ for the normalized linear Lebesgue measure of a set $E$ on the unit circle $\mathbf{T}$. It follows from the classical result of Lusin and Privalov see e.g. [16, that there exist functions $u \in \operatorname{Harm}(\mathbf{D})$ which tend to infinity along almost all radii, while non-tangential growth may occur only on subsets of the unit circle having zero linear measure.

An important generalization of this result is due to Kahane and Katznelson 11. They proved that for any function $v(r), 0<r<1$ such that $v(r) \nearrow \infty$ as $r \nearrow 1$ there exists a function $u \in \operatorname{Harm}(\mathbf{D})$ such that $u\left(r e^{i \theta}\right) \rightarrow \infty$ as $r \nearrow 1$ and also

$$
|u(z)|<v(|z|), z \in \mathbf{D} .
$$

It is known (see [6, 9]), that for a wide class of majorants $v$ realtion (1.1) yields

$$
\liminf _{r \nearrow 1} \frac{u\left(r e^{i \phi}\right)}{v(r)} \leq 0, \text { and } \limsup _{r \nearrow 1} \frac{u\left(r e^{i \phi}\right)}{v(r)} \geq 0
$$

for almost all values of $\phi \in(-\pi, \pi)$. In other words, for almost all $\phi$ the values $u\left(r e^{i \phi}\right)$ "oscillate" between $\pm v(r)$. In this article we study this oscillation in more details.

We restrict ourselves for definiteness to functions which belong to the classical (harmonic) Korenblum class $\mathcal{K}$ consisting of all real functions $u \in$

2000 Mathematics Subject Classification. Primary 31A20; Secondary 60G46, 30J99.

Key words and phrases. Spaces of analytic functions in the disk, Korenblum class, harmonic functions, boundary values, martingales, law of iterated logarithm.

The authors were partly supported by the Research Council of Norway grant 185359/V30 and by ESF grant HCAA. 
$\operatorname{Harm}(\mathbf{D})$ satisfying

$$
u(z) \leq \log \frac{e}{1-|z|}, z \in \mathbf{D} .
$$

This class was introduced and studied in [13]. We refer the reader to [10, 17, 15, 5. for further properties of functions in $\mathcal{K}$, including their behavior near the boundary.

One of the typical examples here is the function

$$
u_{A}(z)=\Re \sum_{n} A^{n} z^{2^{A^{n}}},
$$

where $A \geq 2$ is an integer. It is easy to see that $\left|u_{A}(z)\right| \geq c|\log (1-|z|)|$ on a large portion of the unit disk, and along almost each radius this function oscillates between $c|\log (1-|z|)|$ and $-c \mid \log (1-|z|)$. We give a quantitative description of this oscillation and prove that such oscillation occurs for every harmonic function $u$ such that $u,-u \in \mathcal{K}$.

More precisely for a function $u \in \mathcal{K}$, we consider the weighted average

$$
I_{u}(R, \varphi)=\int_{1 / 2}^{R} \frac{u\left(r e^{i \varphi}\right)}{(1-r)\left(\log \frac{1}{1-r}\right)^{2}} d r, \quad R \in(0,1), \varphi \in(-\pi, \pi) .
$$

A straightforward calculation shows that (1.2) gives

$$
I_{u}(R, \varphi) \lesssim C \log \log \frac{1}{1-R} .
$$

Here and in the sequel we use notation $a \lesssim b$ for the statement: there exists an absolute constant $c$ such that $a \leq c b$; we also write $a \simeq b$ if $a \lesssim b$ and $b \lesssim a$.

If both $u \in \mathcal{K}$ and $-u \in \mathcal{K}$, the behavior of $I_{u}(R, \varphi)$ is controlled by the law of the iterated logarithm:

Theorem 1.1. There exists $K$ such that if $u$ is a harmonic function in $\mathbf{D}$ satisfying

$$
|u(z)| \leq \log \frac{e}{1-|z|}
$$

then

$$
\limsup _{R \nearrow 1} I_{u}(R, \phi)\left(\log \log \frac{1}{1-R} \log _{4} \frac{1}{1-R}\right)^{-1 / 2} \leq K
$$

for almost every $\phi \in(-\pi, \pi]$, (here $\log _{4} x=\log \log \log \log x$ ) and also

$$
\int_{-\pi}^{\pi} I_{u}(R, \phi)^{2} d \phi \leq K \log \log \frac{1}{1-R}
$$

This result is sharp in the following sense: there exists $\alpha>0$ such that the function $u=u_{2}$ defined by (1.3) with $A=2$ satisfies

$$
\limsup _{R \nearrow 1} I_{u}(R, \phi)\left(\log \log \frac{1}{1-R} \log _{4} \frac{1}{(1-R)}\right)^{-1 / 2}>\alpha
$$


for almost every $\phi \in(-\pi, \pi]$.

Theorem 1.1 allows one to obtain more precise estimates of the radial growth for the case when both $u$ and $-u$ belong to $\mathcal{K}$.

Proposition 1.1. Let $u \in \mathcal{K}$ and $-u \in \mathcal{K}$ then for any $a<1 / 2$

$$
\liminf _{r \rightarrow 1} \frac{u\left(r e^{i \phi}\right)(\log |\log (1-r)|)^{a}}{|\log (1-r)|} \leq 0,
$$

for almost every $\phi \in(-\pi, \pi]$.

The proof of Theorem 1.1 is based on approximation of $I_{u}(R, \phi)$ by the sum of discrete martingales and then application of the law of the iterated logarithm for martingales. Our approach is based on the ideas developed by D.L. Burkholder and R.F. Gundy [7], C.Y. Chang, J.M. Wilson, and T.H. Wolf [8], N. Makarov [14], J.M. Anderson, L.D. Pitt [1], and R. Bañuelos, I. Klemes, and C.N. Moore [3], see also the references therein. We also refer the reader to the monographs [2, 4. However approximation of harmonic functions by martingales, which became classical by now, does not yield the desired approximation of the weighted average $I_{u}(R, \varphi)$. In particular the law of the iterated logarithm for trigonometric series does not say much about oscillations of our model function (1.3). Instead we consider the martingale approximation of the function $I_{u}(R, \phi)$ itself. We use "superdyadic" martingales corresponding to the algebras generated by intervals of length $2^{-2^{n}}$ which seem to be more appropriate for our purposes. One of possible ways to think about this construction is to consider a suitable martingale transform of the "classical" martingale of the harmonic function $u$ thinned out to the "super-dyadic" algebras.

These results can be directly applied to lacunary series. We use a result of J.-P. Kahane, M. Weiss, and G. Weiss, [12] in order to describe all functions $u$ which are represented by the series

$$
u(z)=\Re \sum_{k=1}^{\infty} a_{n_{k}} z^{n_{k}}, \quad n_{k+1} / n_{k}>\lambda>1 \text { for each } k,
$$

and belong to $\mathcal{K}$ in terms of the coefficient sequence $\left\{a_{n_{k}}\right\}$. It follows from this description that if a function $u$ of the form (1.10) belongs to $\mathcal{K}$ then $-u \in \mathcal{K}$.

Corollary. Let $u \in \mathcal{K}$ be represented by a lacunary series (1.10). Then (1.6) holds almost everywhere, here $K$ depends only on $\lambda$ from the gap condition and on $C$ in (1.2).

The situation changes drastically when we consider functions $u \in \mathcal{K}$ that satisfy only one-sided estimate (1.2) instead of the two-sided estimate (1.5). This follows from the statement below 
Theorem 1.2. The series

$$
u(z)=\Re \sum_{n>1} 2^{n} \frac{z^{2^{2^{n}}}}{z^{2^{2^{n}}}-1}
$$

converges uniformly on compact sets in $\mathbf{D}$ to a function in $\mathcal{K}$, and, for almost every $\phi \in(-\pi, \pi]$,

$$
\liminf _{R \nearrow 1} I_{u}(R, \phi)\left(\log \log \frac{1}{1-R}\right)^{-1}>0
$$

The article is organized as follows. In the next Section we collect some results on premeasures and (discrete) martingales that are used later. Sections 3 and 4 deal with the martingale approximation of $I_{u}(R, \phi)$ and contain the proof of Theorem 1.1. An example showing that Theorem 1.1] is sharp is given in Section 5, there we also describe harmonic functions in the Korenblum class given by lacunary series. Theorem 1.2 is proved in the last section.

Acknowledgment The main part of this work was done when the authors have been visited the Mathematical Department of University of California, Berkeley. It is our pleasure to thank the Department and Prof. Donald Sarason for kind hospitality.

\section{Preliminaries}

2.1. Representation of functions from $\mathcal{K}$. We refer the reader to the classical article 13 and to the monograph 10 . Let $\mathcal{R}$ be the set of all (open, closed, half-closed) arcs on the unit circle $\mathbf{T}$. A function $\mu: \mathcal{R} \rightarrow \mathbf{R}$ is a premeasure if it satisfies

- $\mu\left(I_{1} \cup I_{2}\right)=\mu\left(I_{1}\right)+\mu\left(I_{2}\right)$ when $I_{1} \cap I_{2}=\emptyset$ and $I_{1} \cup I_{2} \in \mathcal{R}$,

- $\mu(\mathbf{T})=0$

- $\lim _{n \rightarrow \infty} \mu\left(I_{n}\right)=0$ whenever $I_{1} \supset I_{2} \supset \ldots$ and $\cap_{n} I_{n}=\emptyset$.

We say that $\mu$ is $\kappa$-bounded from above, if in addition

$$
\mu(I) \lesssim|I| \log \frac{e}{|I|}
$$

for every $I \in \mathcal{R}$.

Everywhere below we assume (for simplicity) that $u(0)=0$. Let also

$$
P\left(r e^{i \phi}\right)=\frac{1-r^{2}}{\left|e^{i \phi}-r\right|^{2}}
$$

be the standard Poisson kernel. It is proved in [13] that each $u \in \mathcal{K}$ can be represented as the Poisson integral:

$$
u\left(r e^{i \phi}\right)=\int_{-\pi}^{\pi} P\left(r e^{i(\phi-\theta)}\right) d \mu(\theta)
$$


with respect to some $\kappa$-bounded from above premeasure $\mu$. The integral in the right-hand side should be understood as

$$
\int_{-\pi}^{\pi} P\left(r e^{i(\phi-\theta)}\right) d \mu(\theta)=\int_{-\pi}^{\pi}\left(P\left(r e^{i(\phi-\theta)}\right)\right)_{\theta}^{\prime} \mu\left(\left(e^{i \theta}, 0\right)\right) d \theta,
$$

here $\left(e^{i \theta}, 0\right)$ stays for the arc of $\mathbf{T}$ which connects 0 and $e^{i \theta}$.

In the case when $u$ satisfies the two-sided estimate (1.5) the corresponding premeaasure is $\kappa$-bounded from above and below:

$$
|\mu(I)| \lesssim|I| \log \frac{e}{|I|} .
$$

2.2. Martingales. In this part we recall the basic notions and facts about (super-dyadic) martingales (on the unit circle) which will be used in the sequel. We follow mainly [18], see also [19].

Given $n>0$ let $\mathcal{E}_{n}$ be the set of all super-dyadic intervals (on $\mathbf{T}$ ) of length $2^{-2^{n}}$ and $\mathcal{F}_{n}$ be the $\sigma$-algebra generated by $\mathcal{E}_{n}$. Respectively we denote $\mathcal{E}=\cup \mathcal{E}_{n}$. A function $f: \mathbf{T} \rightarrow \mathbf{C}$ is measurable with respect to $\mathcal{F}_{n}$ if it is constant on each $I \in \mathcal{E}_{n}$. Given any $f: \mathbf{T} \rightarrow \mathbf{C}$ we define its expectation with respect to $\mathrm{E}\left(f \mid \mathcal{F}_{n}\right)$ as the measurable (with respect to $\mathcal{F}_{n}$ ) function

$$
\mathrm{E}\left(f \mid \mathcal{F}_{n}\right)=\sum_{I \in \mathcal{E}_{n}}\left(\frac{1}{|I|} \int_{I} f(t) d t\right) \mathbf{1}_{I},
$$

here $\mathbf{1}_{I}$ denotes the characteristic function of $I$. A sequence of function $\left\{f_{n}\right\}, f_{n}: \mathbf{T} \rightarrow \mathbf{C}$ is called a martingale if

- $f_{n}$ is $\mathcal{F}_{n}$ measurable

- $E\left(f_{n} \mid \mathcal{F}_{n-1}\right)=f_{n-1}$

The martingale differences and the square function of a martingale $\left\{f_{n}\right\}$ are defined by

$$
d_{j}=f_{j}-f_{j-1} \text { and } s_{n}=\left(\sum_{j=1}^{n} E\left(d_{j}^{2} \mid \mathcal{F}_{j-1}\right)\right)^{1 / 2},
$$

respectively, see e.g. [18]. Remark, that our martingales are super-dyadic so the formula for $s_{n}$ differs from one usually used for dyadic martingales, we refer the reader to [4] for related discussion. We also denote

$$
u_{n}=\left(2 \log \log s_{n}^{2}\right)^{1 / 2} .
$$

The following statement is a special case of Theorems 1 and 2 in [18.

Theorem B. Let $\mathcal{O}=\{\phi \in(-\pi, \pi]): s_{n}(\phi) \rightarrow \infty$ as $\left.n \rightarrow \infty\right\}$ and $\left|d_{n}\right| \lesssim 1$. Then

$$
\limsup _{n \rightarrow \infty} \frac{f_{n}(\phi)}{s_{n}(\phi) u_{n}(\phi)} \leq 1
$$

for almost all $\phi \in \mathcal{O}$.

We always identify $\phi$ and the point $e^{i \phi} \in \mathbf{T}$. 
Example. Let a premeasure $\mu$ satisfy (2.4). Denote

$$
\begin{gathered}
g_{n}=\sum_{I \in \mathcal{F}_{n}} \mathbf{1}_{I} \frac{\mu(I)}{|I|}, \\
d_{j}=2^{-j}\left(g_{j}-g_{j-1}\right) \text { and } f_{n}=\sum_{j=1}^{n} d_{j} .
\end{gathered}
$$

Then the martingale $\left\{f_{n}\right\}$ meets the conditions of Theorem A.

Remark. The function $u$ in Theorem 1.1 admits representation (2.2), so for each arc $I=\left(e^{i(\theta-\delta)}, e^{i(\theta+\delta)}\right) \subset \mathbf{T}$ the average $|I|^{-1} \mu(I)$ can be considered as an approximation of $u\left((1-\delta) e^{i \theta}\right)$. Taking this into account one can observe that the functions $f_{n}$ from the above example can be viewed as integral sums for $I_{u}\left(1-2^{-2^{n}}, \cdot\right)$. This martingale can be used as a hint in order to guess how Theorem 1.1 should be formulated. However this prove Theorem 1.1 we need a more developed martingale construction.

\section{Atomic DeComposition}

Let a function $u \in \operatorname{Harm}(\mathbf{D})$ satisfy (1.5) and $u(0)=0$. In this section we decompose the function $I_{u}(R, \phi)$ into sum of atoms. Such decomposition (following for example the scheme from [4]) will lead us to a martingale approximation of $I_{u}(R, \phi)$.

\subsection{Preliminary decomposition. Denote}

$$
r_{j}=1-2^{-2^{j}}, j=0,1, \ldots .
$$

Let $r_{n} \leq R<r_{n+1}$. We than have

$$
\begin{aligned}
& I_{u}(R, \phi)=\int_{1 / 2}^{R} \frac{u\left(r e^{i \phi}\right) d r}{(1-r)(\log (1-r))^{2}}= \\
& \sum_{j=1}^{n} \underbrace{\int_{r_{j-1}}^{r_{j}} \frac{u\left(r e^{i \phi}\right)}{(1-r)(\log (1-r))^{2}} d r}_{v_{j}(\phi)}+\int_{r_{n}}^{R} \frac{u\left(r e^{i \phi}\right) d r}{(1-r)(\log (1-r))^{2}}= \\
& =\sum_{j=1}^{n-1} \underbrace{2 v_{j+1}(\phi)-v_{j}(\phi)}_{w_{j}(\phi)}+\underbrace{\left(2 v_{1}(\phi)-v_{n}(\phi)+\int_{r_{n}}^{R} \frac{u\left(r e^{i \phi}\right) d r}{(1-r)|\log (1-r)|^{2}}\right)}_{q_{n}(\phi)} .
\end{aligned}
$$

It follows from (1.5) that $\left|q_{n}(\phi)\right| \lesssim 1$. Therefore in order to prove Theorem 1.1 it suffices to consider

$$
J_{n}(\phi)=\sum_{1}^{n-1} w_{j}(\phi) .
$$

We will approximate the sequence $\left\{J_{n}\right\}$ by the sum of super-dyadic martingales. 
Using representation (2.2) we obtain

$$
v_{j}(\phi)=\int_{-\pi}^{\pi} A_{j}(\phi-\theta) d \mu(\theta), w_{j}(\phi)=\int_{-\pi}^{\pi} B_{j}(\phi-\theta) d \mu(\theta),
$$

where

$$
A_{j}(\psi)=\int_{r_{j-1}}^{r_{j}} \frac{P\left(r e^{i \psi}\right) d r}{(1-r)(\log (1-r))^{2}}, \quad B_{j}(\psi)=2 A_{j}(\psi)-A_{j-1}(\psi) .
$$

Clearly

$$
\int_{-\pi}^{\pi} A_{j}(\phi) d \phi=2^{-j} \text { and } \int_{-\pi}^{\pi} B_{j}(\phi) d \phi=0,
$$

so the kernels $B_{j}$ possess the cancellation property.

3.2. Construction of atoms. The functions $B_{j}(\phi)$ are concentrated mainly in the intervals around zero whose length is of order $2^{-2^{j-4}}$. We approximate them by functions $\tilde{B}_{j}(\phi)$ which are supported in the corresponding intervals. The following (technical) lemma estimates the error of such approximation.

Lemma 3.1. For every $j$ there exists an even function $\tilde{B}_{j}(\psi)$ such that

$$
\begin{gathered}
\operatorname{supp}\left(\tilde{B}_{j}\right) \subset\left(-\frac{1}{64} 2^{-2^{j-4}}, \frac{1}{64} 2^{-2^{j-4}}\right)=: J_{j} \quad \text { and } \quad B_{j}=\tilde{B}_{j} \text { on } \frac{1}{2} J_{j}, \\
\int_{-\pi}^{\pi} \tilde{B}_{j}=\int_{\mathbf{T}} B_{j}=0, \quad\left|\left(B_{j}-\tilde{B}_{j}\right)^{\prime}\right| \lesssim 2^{-2 j} .
\end{gathered}
$$

Moreover, the following estimates hold

$$
\begin{array}{cc}
\text { (a) }\left|\tilde{B}^{\prime}\right| \lesssim 2^{-2 j} 2^{2 \cdot 2^{j}}, & \text { (b) }\left|\tilde{B}^{\prime \prime}\right| \lesssim 2^{-2 j} 2^{3 \cdot 2^{j}}, \quad(c)\left|\tilde{B}_{j}(\alpha) \alpha \log \frac{1}{\alpha}\right| \lesssim 2^{-j} \\
& \int_{-\pi}^{\pi}\left|\tilde{B}_{j}^{\prime}(\alpha) \alpha \log \frac{1}{\alpha}\right| d \alpha \lesssim 1 .
\end{array}
$$

Proof. Let $r_{j}$ be given by (3.1). Elementary calculations show that

$$
A_{j}(\phi) \lesssim \begin{cases}2^{-2 j} 2^{2^{j}}, & \phi \leq 1-r_{j}, \\ 2^{-2 j} \phi^{-1}, & 1-r_{j}<\phi \leq 1-r_{j-1}, \\ 2^{-2 j} \phi^{-2} 2^{-2^{j-1}}, & 1-r_{j-1}<\phi .\end{cases}
$$

Further, we obtain

$$
\left|A_{j}^{\prime}(\phi)\right| \lesssim \begin{cases}2^{-2 j} \phi 2^{3 \cdot 2^{j}}, & \phi \leq 1-r_{j} \\ 2^{-2 j} \phi^{-2}, & 1-r_{j}<\phi \leq 1-r_{j-1} \\ 2^{-2 j} \phi^{-3} 2^{-2^{j-1}}, & 1-r_{j-1}<\phi\end{cases}
$$

and

$$
\left|A_{j}^{\prime \prime}(\phi)\right| \lesssim \begin{cases}2^{-2 j} 2^{3 \cdot 2^{j}}, & \phi \leq 1-r_{j} \\ 2^{-2 j} \phi^{-3}, & 1-r_{j}<\phi \leq 1-r_{j-1} \\ 2^{-2 j} \phi^{-4} 2^{-2^{j-1}}, & 1-r_{j-1}<\phi\end{cases}
$$


Let $\alpha$ and $\beta$ be even smooth functions, $0 \leq \alpha \leq 1$, such that

$$
\begin{gathered}
\alpha=1, \quad \text { on }\left(-2^{-7} 2^{-2^{j-4}}, 2^{-7} 2^{-2^{j-4}}\right) ; \\
\operatorname{supp}(\alpha) \subset\left[-2^{-6} 2^{-2^{j-4}}, 2^{-6} 2^{-2^{j-4}}\right], \quad\left|\alpha^{\prime}\right| \lesssim 2^{2^{j-4}}, \text { and }\left|\alpha^{\prime \prime}\right| \lesssim 2^{2^{j-3}} ; \\
\int_{-\pi}^{\pi} \beta=1, \quad \operatorname{supp}(\beta) \subset\left[-2^{-6} 2^{-2^{j-4}},-2^{-7} 2^{-2^{j-4}}\right] \cup\left[2^{-7} 2^{-2^{j-4}}, 2^{-7} 2^{-2^{j-4}}\right] ; \\
|\beta| \lesssim 2^{2^{j-4}}, \quad\left|\beta^{\prime}\right| \lesssim 2^{2^{j-3}}, \quad \text { and }\left|\beta^{\prime \prime}\right| \lesssim 2^{2^{j-2}} .
\end{gathered}
$$

Then we define

$$
\tilde{B}_{j}=\alpha B_{j}+\beta \int_{-\pi}^{\pi}\left(B_{j}-\alpha A_{j}\right)
$$

Clearly $\tilde{B}_{j}$ is an even function. The required estimates now follows by a direct inspection.

Now let

$$
\tilde{w}_{j}(\phi)=\int_{-\pi}^{\pi} \tilde{B}_{j}(\phi-\theta) d \mu(\theta) .
$$

Lemma 3.2. The following inequality holds

$$
\left|w_{j}(\phi)-\tilde{w}_{j}(\phi)\right| \lesssim 2^{-2 j}
$$

for any $j$ and $\phi \in(-\pi, \pi)$.

Proof. Clearly (1.5) implies that $|\mu(I)| \lesssim 1$ for any interval $I$ on the circle. Since $B_{j}-\tilde{B}_{j}$ is an even function and $\mu(\mathbf{T})=0$ we have

$$
\begin{gathered}
\left|w_{j}(\phi)-\tilde{w}_{j}(\phi)\right|=\left|\int_{-\pi}^{\pi}\left(B_{j}-\tilde{B}_{j}\right)(\phi-\theta) d \mu(\theta)\right| \\
=\left|\int_{0}^{\pi}\left(B_{j}-\tilde{B}_{j}\right)^{\prime}(\theta) \mu(\phi-\theta, \phi+\theta)\right| \lesssim 2^{-2 j} .
\end{gathered}
$$

This lemma implies

$$
\left|\sum_{1}^{n} w_{j}(\phi)-\sum_{1}^{n} \tilde{w}_{j}(\phi)\right| \lesssim 1
$$

We divide the circle into $64 \cdot 2^{2^{k}}$ non-overlapping arcs of length $\frac{1}{64} 2^{-2^{k}}$ and define by $\mathcal{H}_{k}$ the collection of these arcs. Let also $\mathcal{H}=\cup_{k} \mathcal{H}_{k}$. We obtain (3.11)

$$
\tilde{w}_{j}(\phi)=\int_{-\pi}^{\pi} \tilde{B}_{j}(\phi-\theta) d \mu(\theta)=\sum_{I \in \mathcal{H}_{j-4}} \int_{I} \tilde{B}_{j}(\phi-\theta) d \mu(\theta)=\sum_{I \in \mathcal{H}_{j-4}} \lambda_{I}(\phi) .
$$

Functions $\lambda_{I}$ are our atoms. For each $I \in \mathcal{H}_{k}, k>6$ we have

$$
\operatorname{supp} \lambda_{I} \subset 3 I \text {; }
$$


and

$$
\int_{3 I} \lambda_{I}=\int_{-\pi}^{\pi} \lambda_{I}(\phi) d \phi=\int_{-\pi}^{\pi} \int_{I} \tilde{B}_{j}(\phi-\theta) d \mu(\theta) d \phi=0 .
$$

4. From atoms to martingales

4.1. Construction of martingales. Relation (3.10) gives a decomposition of $\sum_{j=1}^{n} \tilde{w}_{j}$ into the sum of atoms $\lambda_{I}, I \in \mathcal{H}$. It may happen that $\operatorname{supp} \lambda_{I_{1}} \cap$ $\operatorname{supp} \lambda_{I_{2}} \neq \emptyset$ for (neighboring) $I_{1}, I_{2} \in \mathcal{H},\left|I_{1}\right|=\left|I_{2}\right|$.

Given $\omega \in \mathbf{T}$ and an arc $I \in \mathbf{T}$ we denote $\omega I=\{\omega \zeta ; \zeta \in I\}$. Respectively $\omega \mathcal{E}=\{\omega I ; I \in \mathcal{E}\}, \omega \mathcal{E}_{n}=\left\{\omega I ; I \in \mathcal{E}_{n}\right\}$. The lemma below follows from a more general statement Lemma 2.1.2 in [4], see also [8] (we adjust the formulation for our setting.)

Lemma 4.1. There exists a finite partition $\mathcal{H}=\cup_{s=1}^{N} V^{(s)}$ and a set of points $\left\{\omega_{s}\right\}_{s=1}^{N} \subset \mathbf{T}$ such that $V^{(s)} \cap V^{(t)}=\emptyset, s \neq t$ and, for each $I \in V^{(s)} \cap \mathcal{H}_{k}$, there exists $I^{\prime} \in \omega_{s} \mathcal{E}_{k}$ for which $\operatorname{supp} \lambda_{I} \subset 3 I \subset I^{\prime}$. In addition if, for some $s$ and $k, I_{1}, I_{2} \in V^{(s)} \cap \mathcal{H}_{k}, I_{1} \neq I_{2}$ then $I_{1}^{\prime} \cap I_{2}^{\prime}=\emptyset$.

Now for each $s=1,2, \ldots, N$ and $n \geq 1$ we define

$$
\Lambda_{n}^{(s)}(\phi)=\sum_{I \in V^{(s)}, 64|I| \geq 2^{-2^{n}}} \lambda_{I}(\phi) .
$$

Then

$$
\sum_{j=1}^{n} \tilde{w}_{j}(\phi)=\sum_{s=1}^{N} \Lambda_{n-4}^{(s)}(\phi) .
$$

We consider the corresponding martingales with the sequence of (shifted) super-dyadic $\sigma$-algebras

$$
f_{n}^{(s)}=E\left(\Lambda_{n}^{s} \mid \omega_{s} \mathcal{F}_{n}\right)
$$

4.2. Estimate of the martingale approximation. Our first aim is to estimate the error

$$
\left|f_{n}^{(s)}-\Lambda_{n}^{(s)}\right| \leq \sum_{I \in V^{s}, 64|I| \geq 2^{-2^{n}}}\left|E\left(\lambda_{I} \mid \omega_{s} \mathcal{F}_{n}\right)-\lambda_{I}\right|
$$

Lemma 4.2. The following inequality holds

$$
\left|f_{n}^{(s)}(\phi)-\Lambda_{n}^{(s)}(\phi)\right| \lesssim 1
$$

Proof. First we prove that

$$
\left|\lambda_{I}(\phi)\right| \lesssim 1 \quad \text { and } \quad\left|\lambda_{I}^{\prime}(\phi)\right| \leq|I|^{-64}
$$

for any $I \in \mathcal{H}$ and any $\phi \in(-\pi, \pi]$;

Indeed let $\omega_{\phi}(\theta)=\int_{\phi}^{\theta} d \mu=\mu(\phi, \theta)$ and let $I=(\alpha, \beta) \in \mathcal{H}_{j-4}$. We have

$$
\left|\lambda_{I}(\phi)\right|=\left|\int_{I} \tilde{B}_{j}(\phi-\theta) d \mu(\theta)\right| \leq
$$




$$
\left|\int_{I} \tilde{B}_{j}^{\prime}(\phi-\theta) \omega_{\phi}(\theta) d \theta\right|+\left|\tilde{B}_{j}(\phi-\alpha) \omega_{\phi}(\alpha)\right|+\left|\tilde{B}_{j}(\phi-\beta) \omega_{\phi}(\beta)\right| .
$$

Using inequalities (3.8) and (3.7 (c)), we obtain the first estimate in (4.5) To prove the second estimate we write

$$
\left|\lambda_{I}^{\prime}(\phi)\right| \leq\left|\int_{I} \tilde{B}_{j}^{\prime \prime}(\phi-\theta) \omega_{\phi}(\theta) d \theta\right|+\left|\tilde{B}_{j}^{\prime}(\phi-\alpha) \omega_{\phi}(\alpha)\right|+\left|\tilde{B}_{j}^{\prime}(\phi-\beta) \omega_{\phi}(\beta)\right|
$$

and use the inequalities (3.7 (a)) and (3.7(b)).

Now we have

$$
\left|E\left(\lambda_{I} \mid \omega_{s} \mathcal{F}_{n}\right)-\lambda_{I}\right| \leq 2^{-2^{n}} \max \left|\lambda_{I}^{\prime}\right|
$$

This inequality together with (4.5) imply

$$
\left|f_{n}^{(s)}(\phi)-\Lambda_{n}^{(s)}(\phi)\right| \leq \sum_{k=1}^{n} \sum_{I \in V^{s} \cap \mathcal{H}_{k}, I^{\prime} \ni \phi}\left|E\left(\lambda_{I} \mid \omega_{s} \mathcal{F}_{n}\right)(\phi)-\lambda_{I}(\phi)\right| \leq
$$

$$
\sum_{k=1}^{n-6} \sum_{I \in V^{s} \cap \mathcal{H}_{k}, I^{\prime} \ni \phi}\left|E\left(\lambda_{I} \mid \omega_{s} \mathcal{F}_{n}\right)(\phi)-\lambda_{I}(\phi)\right|+
$$

$$
\sum_{k=n-5}^{n} \sum_{I \in V^{s} \cap \mathcal{H}_{k}, I^{\prime} \ni \phi}\left(\left|E\left(\lambda_{I} \mid \omega_{s} \mathcal{F}_{n}\right)(\phi)\right|+\left|\lambda_{I}(\phi)\right|\right) \lesssim 2^{-2^{n}} \sum_{k=1}^{n-6} 2^{2^{k+6}}+1 \lesssim 1,
$$

and (4.4) now follows.

For each martingale $\left\{f_{n}^{(s)}\right\}$ we can now estimate its square function,

$$
s_{n}^{(s)}=\left(\sum_{k=1}^{n} E\left(\left|f_{k}^{(s)}-f_{k+1}^{(s)}\right|^{2} \mid \mathcal{F}_{k}\right)\right)^{1 / 2} .
$$

Lemma 4.3. For each $s=1, \ldots, N$ and $n \geq 1$

$$
\left|f_{n}^{(s)}-f_{n+1}^{(s)}\right| \lesssim 1 \quad \text { and } \quad s_{n}^{(s)} \lesssim \sqrt{n}
$$

Proof. The first inequality follows from Lemma 4.2 and (4.5). The second inequality is now straightforward.

4.3. Mean estimates. We first prove inequality (1.7) from Theorem 1.1. namely

$$
\int_{-\pi}^{\pi} I_{u}(R, \phi)^{2} d \phi \lesssim \log \log \frac{1}{1-R} .
$$

Let, as before, $r_{n}$ 's be given by (3.1) and $r_{n} \leq R<r_{n+1}$. Then (see (3.2), (3.10), (3.5), and (4.4) )

$$
\left|I_{u}(R, \phi)-\sum_{s=1}^{N} f_{n-4}^{(s)}(\phi)\right| \lesssim 1
$$


Therefore it suffices to prove that

$$
\int_{-\pi}^{\pi} f_{n-4}^{(s)}(\phi)^{2} d \phi \lesssim n \simeq \log \log \frac{1}{1-R}
$$

for each $s=1,2, \ldots, N$.

We use the fact that the martingale differences $f_{j}^{(s)}(\phi)-f_{j-1}^{(s)}(\phi)$ are pairwise orthogonal. Therefore

$$
\begin{gathered}
\int_{\mathbf{T}}\left|f_{n-4}^{(s)}(\phi)\right|^{2} d \phi=\int_{\mathbf{T}}\left|\sum_{j=1}^{n-4}\left(f_{j}^{(s)}(\phi)-f_{j-1}^{(s)}(\phi)\right)\right|^{2} d \phi= \\
\int_{\mathbf{T}} \sum_{j=1}^{n-4}\left|f_{j}^{(s)}(\phi)-f_{j-1}^{(s)}(\phi)\right|^{2} d \phi=\int_{\mathbf{T}}\left(s_{n-4}^{(s)}(\phi)\right)^{2} d \phi \lesssim n,
\end{gathered}
$$

the last inequality follows from (4.7). This yields (1.7).

4.4. The law of the iterated logarithm. The relation (1.6) from Theorem 1.1 now follows easily from Theorem A and the estimate (4.7) of the square functon. Indeed, since $\left|I_{u}(R, \phi)-\sum_{s=1}^{N} f_{n-4}^{(s)}(\phi)\right| \lesssim 1$ it suffices to prove that for each $s$

$$
\limsup _{n} \frac{f_{n}^{(s)}}{n \log \log n} \lesssim 1
$$

almost everywhere. In case $s_{n}^{(s)}(\phi) \rightarrow \infty$ this follows from relations (2.8) and (4.7). Otherwise $s_{n}^{(s)}(\phi)$ stays bounded and

$$
\left|f_{n}^{(s)}(\phi)\right| \leq \sum_{j=1}^{n}\left|f_{j}^{(s)}(\phi)-f_{j-1}^{(s)}(\phi)\right| \leq n^{1 / 2} s_{n}^{(s)}(\phi)^{1 / 2} .
$$

This completes the proof of Theorem 1.1. Proposition 1.1 formulated in the introduction follows readily.

\section{LACUNARY SERIES}

5.1. Example. We begin with an example showing that Theorem 1.1 is sharp. Let

$$
u(z)=\Re \sum_{n=1}^{\infty} 2^{n} z^{2^{2^{n}}}, \quad \text { and } \quad I_{u}(R, \phi)=\int_{1 / 2}^{R} \frac{u\left(r e^{i \phi}\right)}{(1-r)(\log (1-r))^{2}} d r .
$$

It is proved in [6] that $u \in \mathcal{K}$. We will show that for some $a>0$

$$
\limsup _{R \rightarrow 1} I_{u}(R, \phi)\left(\log \log \frac{1}{1-R} \log _{4} \frac{1}{(1-R)}\right)^{-1 / 2} \geq a
$$

for almost all $\phi \in(-\pi, \pi]$. Let

$$
c_{n}=2^{n} \int_{1 / 2}^{1} \frac{r^{2^{2^{n}}}}{(1-r)(\log (1-r))^{2}} d r
$$


and $r_{k}=1-2^{-2^{k}}$. Suppose that $R \in\left(r_{k}, r_{k+1}\right)$. We prove first that

$$
\left|I_{u}(R, \phi)-\sum_{j=1}^{k} c_{j} \cos \left(2^{2^{j}} \phi\right)\right| \lesssim 1
$$

Indeed,

$$
\left|I_{u}(R, \phi)-I_{u}\left(r_{k}, \phi\right)\right| \lesssim \int_{r_{k}}^{r_{k+1}} \frac{d r}{(1-r)|\log (1-r)|} \lesssim 1
$$

Further,

$$
I_{u}\left(r_{k}, \phi\right)=\sum_{j=1}^{\infty} \int_{1 / 2}^{r_{k}} \frac{2^{j} r^{2^{2^{j}}}}{(1-r)(\log (1-r))^{2}} d r \cos \left(2^{2^{j}} \phi\right)=\sum_{j=1}^{\infty} c_{j, k} \cos \left(2^{2^{j}} \phi\right) .
$$

For $j>k$ we have

$$
c_{j, k}=\int_{1 / 2}^{r_{k}} \frac{2^{j} r^{2^{2^{j}}}}{(1-r)(\log (1-r))^{2}} d r \lesssim 2^{j}\left(1-2^{-2^{k}}\right)^{2^{2^{j}}} \lesssim 2^{j} \exp \left(-2^{2^{j}-2^{k}}\right)
$$

and $\sum_{j>k} c_{j, k} \lesssim 1$. Finally, for $j \leq k$ we get

$\left|c_{j, k}-c_{j}\right|=2^{j} \int_{r_{k}}^{1} \frac{r^{2^{2^{j}}}}{(1-r)(\log (1-r))^{2}} d r \leq 2^{j} \int_{r_{k}}^{1} \frac{d r}{(1-r)(\log (1-r))^{2}} \lesssim 2^{j-k}$.

Therefore $\sum_{j \leq k}\left|c_{j, k}-c_{j}\right| \lesssim 1$. Inequality (5.1) is proved.

Now we apply the law of the iterated logarithm proved in [20] to the lacunary series

$$
\sum_{j} c_{j} \cos \left(2^{2^{j}} \phi\right)
$$

First note that

$$
\begin{aligned}
c_{j}= & 2^{j} \int_{1 / 2}^{1} \frac{r^{2^{2^{j}}}}{(1-r)(\log (1-r))^{2}} d r \leq 2^{j} \int_{1 / 2}^{r_{j-1}} \frac{r^{2^{2^{j}}}}{(1-r)(\log (1-r))^{2}} d r+ \\
& 2^{j} \int_{r_{j-1}}^{1} \frac{d r}{(1-r)(\log (1-r))^{2}} \lesssim 2^{j}\left(1-2^{-2^{j-1}}\right)^{2^{2^{j}}}+2^{j} 2^{-j+1} \lesssim 1 .
\end{aligned}
$$

and

$$
c_{j} \geq 2^{j} r_{j}^{2^{2^{j}}} \int_{r_{j}}^{1} \frac{d r}{(1-r)(\log (1-r))^{2}} \gtrsim 1 .
$$

Thus the coefficients in (5.3) are bounded from 0 and $\infty$ and

$$
B_{k}^{2}=\frac{1}{2} \sum_{j=1}^{k} c_{j}^{2} \simeq k
$$


Finally

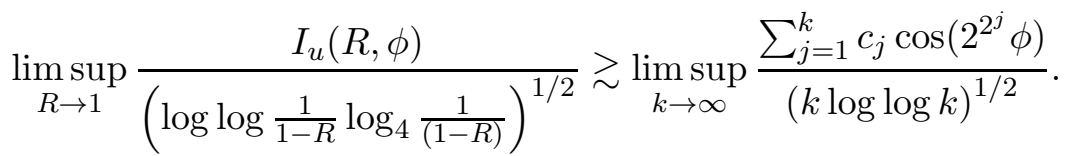

The last upper limit is larger than a constant for almost all $\phi \in(-\pi, \pi]$ by the law of the iterated logarithm for trigonometric lacunary series.

5.2. Description of Korenblum harmonic functions represented by lacunary series. Using results from [13] and [12], we get the following description of lacunary series that represent Korenblum functions.

Proposition 5.1. Let $\left\{n_{k}\right\}_{k=1}^{\infty}$ be a sequence of positive integers such that $n_{k+1} \geq \lambda n_{k}$ for each $k$, where $\lambda>1$. Let

$$
u(z)=\Re \sum_{k} c_{n_{k}} z^{n_{k}}, \quad c_{n_{k}} \in \mathbf{C},
$$

where the series converges in the unit disc. Then the following conditions are equivalent:

(1) there exists $\gamma_{1}$ such that $u(z) \leq \gamma_{1} \log \frac{e}{1-|z|}$ for any $z \in \mathbf{D}$;

(2) there exists $\gamma_{2}$ such that $|u(z)| \leq \gamma_{2} \log \frac{e}{1-|z|}$ for any $z \in \mathbf{D}$;

(3) there exists $\gamma_{3}$ such that $\sum_{n_{k} \leq N}\left|c_{n_{k}}\right| \leq \gamma_{3} \log N$ for any $N \geq 2$.

Proof. Let $u_{r}\left(e^{i \phi}\right)=u\left(r e^{i \phi}\right)$. We show first that (1) implies (3). It follows from [13, p.209] that $\left|c_{n_{k}}\right| \leq C_{1} \log n_{k}$, where $C_{1}=C_{1}\left(\gamma_{1}\right)$.

Let $r_{N}$ be such that $r_{N}^{N}=1 / 2$, we have

$$
u\left(r_{N} e^{i \phi}\right)=\Re \sum_{n_{k} \leq N} c_{n_{k}} r_{N}^{n_{k}} e^{i n_{k} \phi}+\Re \sum_{n_{k}>N} c_{n_{k}} r_{N}^{n_{k}} e^{i n_{k} \phi}=s_{N}(\phi)+t_{N}(\phi) .
$$

The reminder term can be estimated as follows

$$
\left|t_{N}(\phi)\right| \leq C_{1} \sum_{n_{k}>N} \log n_{k} 2^{-n_{k} / N} \leq \beta\left(\gamma_{1}, \lambda\right) \log N
$$

By Theorem I in [12] there exists $\alpha=\alpha(\lambda)$ and $\phi \in(-\pi, \pi)$ such that

$$
s_{N}(\phi) \geq \alpha \sum_{n_{k} \leq N}\left|c_{n_{k}} r_{N}^{n_{k}}\right| .
$$

(This statement is elementary when $\lambda>2$; the result in [12] is more general.) Thus we have

$$
\sum_{n_{k} \leq N}\left|a_{n_{k}}\right| \leq 2 \sum_{n_{k} \leq N}\left|c_{n_{k}} r_{N}^{n_{k}}\right| \leq 2 \alpha^{-1}\left(u\left(r e^{i \phi}\right)+\left|t_{N}(\phi)\right|\right) \leq \gamma_{3} \log N .
$$

Now assume that (3) holds and let $r_{N}<r \leq r_{N+1}$. Then (2) follows readily

$$
\left|u\left(r e^{i \phi}\right)\right| \leq \sum_{n_{k} \leq N}\left|c_{n_{k}}\right|+\sum_{n_{k}>N} \gamma_{3} \log n_{k} r^{n_{k}} \leq \gamma_{2} \log \frac{e}{1-r} .
$$


Thus the Korenblum functions represented by (5.4) satisfy the assumption of Theorem 1.1.

\section{Non-Oscillation: ExAmple}

6.1. Construction. In this section we prove Theorem 1.2 ,

Lemma 6.1. The series

$$
u(z)=\Re \sum_{n \geq 1} 2^{n} \frac{z^{2^{2^{n}}}}{z^{2^{2^{n}}}-1}=\Re \sum_{n \geq 1} 2^{n} a_{n}(z)
$$

converges uniformly on compact sets in $\mathbf{D}$ to a function in $\mathcal{K}$.

Proof. We split the unit disk into disjoint annuli

$$
\mathbf{D}=\{z:|z|<1 / 2\} \cup\left(\cup_{n} A_{n}\right), A_{n}=\left\{z: 2^{-2^{n}} \geq 1-|z|>2^{-2^{n+1}}\right\} .
$$

Let $z \in A_{n}$ and $k>n+1$. Then (by a straightforward calculation)

$$
\left|\frac{2^{k+1} a_{k+1}(z)}{2^{k} a_{k}(z)}\right| \lesssim 2^{-2^{2^{k}}}
$$

Choosing $n$ sufficiently large we will see that the ratio of two consequent terms with numbers larger than $n$ does not exceed some $q<1$. This yields the uniform convergence of (6.1) on compact sets in $\mathbf{D}$.

A direct estimate shows that $\left|a_{n+1}(z)\right| \leq C$ when $z \in A_{n}$ and thus

$$
\sum_{k>n} 2^{k}\left|a_{k}(z)\right| \leq C_{q} \log \frac{1}{1-|z|}, z \in A_{n} .
$$

To complete the proof it suffices to estimate $\Re \sum_{k \leq n} 2^{k} a_{k}(z)$. Since

$$
\Re(w /(w-1)) \leq 1 / 2 \text { for } w \in \mathbf{D}
$$

we obtain:

$$
\Re \sum_{k \leq n} 2^{k} a_{k}(z) \leq \sum_{k \leq n} 2^{k} \leq C \log \frac{1}{1-|z|}, z \in A_{n} .
$$

6.2. Remark. Since $u(0)=0$ we have $\int_{-\pi}^{\pi} u\left(r e^{i \theta}\right) d \theta=0$ for all $r \in(0,1)$. The summands in (6.1) have the form

$$
a_{n}(z)=\Phi\left(z^{2^{2^{n}}}\right) ; \Phi(w)=\frac{w}{w-1}, w \in \mathbf{D} .
$$

The function $\zeta=\Phi(w)$ maps the unit disk $\mathbf{D}$ onto the half-plane $\{\zeta ; \Re \zeta<$ $1 / 2\}$. The asymmetry in the distribution of $\Re \Phi(w)$ forces $u$ to attain huge negative values on "small" parts of $\mathbf{D}$ while being positive (or slightly negative) on the remaining part of $\mathbf{D}$ thus maintaining zero average along the circles $|z|=r$. In this construction the oscillation along almost all radii disappears. 
6.3. Main lemma. Let for brevity $N_{n}=2^{2^{n}}$ so that $N_{n+1}=N_{n}^{2}$. Fix some $a>2$ and let

$$
\begin{gathered}
E_{n}=\left\{\phi \in(-\pi, \pi]: \cos N_{n} \phi>1-n^{-a}\right\}, \\
E_{n, m}=\left\{\phi \in(-\pi, \pi]: \cos N_{n} \phi>1-N_{m}^{-1}\right\}, \\
F_{m}=\left(\cup_{n \geq m} E_{n}\right) \cup\left(\cup_{n<m} E_{n, m}\right), \quad \text { and } \quad F=\cap_{m} F_{m} .
\end{gathered}
$$

Since $\left|E_{n}\right| \simeq n^{-a / 2}$ and $\sum_{1 \leq n<m}\left|E_{n, m}\right| \rightarrow 0(m \rightarrow \infty)$, we have $|F|=0$. Theorem 1.2 now follows from the lemma below:

Lemma 6.2. For each $\phi \notin F$

$$
\liminf _{R \nearrow 1} \frac{I_{u}(R, \phi)}{\log \log \frac{1}{1-R}}>0 .
$$

Proof. We fix $\phi \notin F$. There exists $m$ such that $\phi \notin F_{m}$.

For each $l$ we denote

$$
I_{l}(\phi)=\int_{1-N_{l}^{-1}}^{1-N_{l}^{-2}} \frac{u\left(r e^{i \phi}\right)}{(1-r)\left(\log \frac{1}{1-r}\right)^{2}} d r
$$

Assume $R=1-2^{-2^{t}}, t>m$, and let $\lfloor t\rfloor$ stay for the integer part of $t$. We then have

$$
I_{u}(R, \phi)=\sum_{l<\lfloor t\rfloor} I_{l}(\phi)+\int_{1-N_{\lfloor t\rfloor}^{-1}}^{R} \frac{u\left(r e^{i \phi}\right)}{(1-r)\left(\log \frac{1}{1-r}\right)^{2}} d r .
$$

We will prove that there exist constants $\gamma, \Gamma>0$ and a number $l_{0}>m$ such that, for $l>l_{0}$

$$
I_{l}(\phi)>\gamma
$$

and

$$
\int_{1-N_{\lfloor t\rfloor}^{-1}}^{R} \frac{u\left(r e^{i \phi}\right)}{(1-r)\left(\log \frac{1}{1-r}\right)^{2}} d r>-\Gamma, \text { when } t>l_{0} .
$$

Then (6.4) will follow readily.

\subsection{Technical details. We have}

$$
I_{l}(\phi)=\sum_{j=1}^{\infty} \Re \int_{1-N_{l}^{-1}}^{1-N_{l}^{-2}} 2^{j} \frac{\left(r e^{i \phi}\right)^{N_{j}}}{\left(r e^{i \phi}\right)^{N_{j}}-1} \frac{d r}{(1-r)\left(\log \frac{1}{1-r}\right)^{2}}=\sum_{j=1}^{\infty} i_{l, j}(\phi),
$$

and similarly

$$
\int_{1-N_{\lfloor t\rfloor}^{-1}}^{R} \frac{u\left(r e^{i \phi}\right)}{(1-r)\left(\log \frac{1}{1-r}\right)^{2}} d r=\sum_{j=1}^{\infty} \tilde{i}_{j}(\phi) .
$$

Inequalities (6.6) and (6.7) follow from the propositions below. 
Proposition 6.1. Let $\phi \notin F_{m}$. There exist $\gamma>0$ and $l_{0}>m$ such that for all $l>l_{0}$

$$
\begin{gathered}
i_{l, j}(\phi)>0, \text { if } j<l, \\
i_{l, l}(\phi)>2 \gamma
\end{gathered}
$$

and

$$
\sum_{j>l}\left|i_{l, j}(\phi)\right|<\gamma
$$

Proposition 6.2. Let $\phi \notin F_{m}$. There exist $\Gamma>0$ and $l_{0}>m$ such that for $\lfloor t\rfloor>l_{0}$

$$
\begin{gathered}
\tilde{i}_{j}(\phi)>0 \text { if } j<\lfloor t\rfloor, \\
\tilde{i}_{\lfloor t\rfloor}>-\Gamma / 2,
\end{gathered}
$$

and

$$
\sum_{j>\lfloor t\rfloor}\left|\tilde{i}_{j}(\phi)\right|<\Gamma / 2, \text { if } l>l_{0} .
$$

Proof of (6.8) and (6.11). Denote $c_{j}(\phi)=\cos N_{j} \phi$. We have

$$
i_{l, j}(\phi)=\int_{1-N_{l}^{-1}}^{1-N_{l}^{-2}} 2^{j} \frac{r^{N_{j}}\left(r^{N_{j}}-c_{j}(\phi)\right)}{r^{2 N_{j}}-2 r^{N_{j}} c_{j}(\phi)+1} \frac{d r}{(1-r)\left(\log \frac{1}{1-r}\right)^{2}} .
$$

Then, for $r>1-N_{l}^{-1}$, and $m<j<l$ we obtain

$$
r^{N_{j}} \geq\left(1-N_{j}^{-2}\right)^{N_{j}}>1-2^{-2^{j}}>1-j^{-a}>c_{j}(\phi), \text { when } \phi \notin F_{m} .
$$

For $j \leq m<l$ we have

$$
r^{N_{j}} \geq\left(1-N_{m}^{-2}\right)^{N_{j}}>1-N_{m}^{-1}>c_{j}(\phi)
$$

for any $\phi \notin F_{m}$. Hence for all $j<l$ we have $\Re a_{j}\left(r e^{i \phi}\right)>0$ when $r>1-N_{l}^{-1}$, and $\phi \notin F_{m}$, which implies both (6.8) for all $l>m$ and (6.11).

Proof of (6.9). We fix $l>m$ and choose $r^{*} \in\left(1-N_{l}^{-1}, 1-N_{l}^{-2}\right)$ so that

$$
\left(r^{*}\right)^{N_{l}}=1-\frac{1}{2} l^{-a} .
$$

For $\phi \notin F_{m}$ we have $c_{l}(\phi)<c_{l}:=1-l^{-a}$ and

$$
\begin{gathered}
i_{l, l}(\phi) \geq \int_{1-N_{l}^{-1}}^{1-N_{l}^{-2}} 2^{l} \frac{r^{N_{l}}\left(r^{N_{l}}-c_{l}\right)}{r^{2 N_{l}-2 r^{N_{l}} c_{l}+1} \frac{d r}{(1-r)\left(\log \frac{1}{1-r}\right)^{2}}=} \\
\left(\int_{1-N_{l}^{-1}}^{r^{*}}+\int_{r^{*}}^{1-N_{l}^{-2}}\right) 2^{l} \frac{r^{N_{l}}\left(r^{N_{l}}-c_{l}\right)}{\left(r^{\left.N_{l}-c_{l}\right)^{2}+1-c_{l}^{2}} \frac{d r}{(1-r)\left(\log \frac{1}{1-r}\right)^{2}}\right.} \\
=j_{1}+j_{2},
\end{gathered}
$$


Applying the inequality $x\left(x^{2}+y^{2}\right)^{-1} \geq-(2 y)^{-1}$, we have

$$
\frac{r^{N_{l}}-c_{l}}{\left(r^{N_{l}}-c_{l}\right)^{2}+\left(1-c_{l}^{2}\right)} \geq-l^{a / 2}
$$

We also have $\log 1 /(1-r) \simeq 2^{l}$, for $r \in\left(1-N_{l}^{-1}, 1-N_{l}^{-2}\right)$. Therefore

$$
j_{1} \gtrsim-2^{-l} l^{a / 2} \int_{1-N_{l}^{-1}}^{r^{*}} \frac{d r}{1-r} \gtrsim-2^{-l} l^{a / 2} a \log l .
$$

The right-hand side here can be made arbitrary small by choosing sufficiently large $l_{0}$.

To complete the proof of $(6.9)$ it remains to show that

$$
j_{2} \simeq 1
$$

For $r>r^{*}$ and $c_{l}(\phi)<c_{l}$ we have

$$
1 \geq \frac{r^{N_{l}}\left(r^{N_{l}}-c_{l}(\phi)\right)}{r^{2 N_{l}}-2 r^{N_{l}} c_{l}(\phi)+1}>\frac{1-\frac{1}{2} l^{-a}}{4-\frac{3}{2} l^{-a}}>\frac{1}{4} .
$$

Hence

$$
j_{2} \approx 2^{-l} \int_{r^{*}}^{1-N_{l}^{-2}} \frac{d r}{(1-r)} .
$$

Relation (6.15) now follows from

$$
2^{-l} \int_{1-N_{l}^{-1}}^{1-N_{l}^{-2}} \frac{d r}{(1-r)}=\frac{\log 2}{2} ; \quad 2^{-l} \int_{1-N_{l}^{-1}}^{r^{*}} \frac{d r}{(1-r)} \rightarrow 0, \text { as } l \rightarrow \infty .
$$

Proof of (6.12) can be done in a similar way if one chooses $r^{*}$ so that $\left(r^{*}\right)^{N\lfloor t\rfloor}=1-\frac{1}{2}\lfloor t\rfloor^{-a}$ and repeats the above reasonings.

Proof of (6.10) and (6.13). These inequalities can be proved in the same manner so we restrict ourselves to (6.10) only.

First we estimate the sum $\sum_{j>l+1}\left|i_{l, j}(\phi)\right|$. Inequality (6.2) implies that for $l>l_{0}$

$$
\sum_{j>l+1}\left|i_{l, j}(\phi)\right| \lesssim 2^{-l}
$$

and the contribution of this sum can be done arbitrary small by choosing $l_{0}$ large enough.

It remains to estimate

$$
i_{l, l+1}(\phi)=\int_{1-N_{l}^{-1}}^{1-N_{l}^{-2}} 2^{l+1} \frac{r^{N_{l+1}}\left(r^{N_{l+1}}-c_{l+1}(\phi)\right)}{r^{2 N_{l+1}}-2 r^{N_{l+1}} c_{l+1}(\phi)+1} \frac{d r}{(1-r)\left(\log \frac{1}{1-r}\right)^{2}} .
$$


Since $r^{N_{l+1}}<2^{-1}$ for $r \in\left(1-N_{l}^{-1}, 1-N_{l}^{-2}\right)$ we have

$$
\begin{aligned}
\left|i_{l, l+1}(\phi)\right| \lesssim 2^{-l} \int_{1-N_{l}^{-1}}^{1-N_{l}^{-2}} \frac{r^{N_{l+1}}}{1-r} d r & = \\
& 2^{-l} \int_{1-N_{l}^{-1}}^{\rho_{l}} \frac{r^{N_{l+1}} d r}{1-r}+2^{-l} \int_{\rho_{l}}^{1-N_{l}^{-2}} \frac{r^{N_{l+1}} d r}{1-r},
\end{aligned}
$$

here $\rho_{l}=1-\log \frac{1}{\varepsilon} N_{l}^{-2} \in\left(1-N_{l}^{-1}, 1-N_{l}^{-2}\right)$. Then for all sufficiently large $l$ we obtain $r^{N_{l+1}}<2 \varepsilon$ when $r \in\left(1-N_{l}^{-1}, \rho_{l}\right)$ and thus the first integral is less than const $\varepsilon$. Finally,

$$
2^{-l} \int_{\rho_{l}}^{1-N_{l}^{-2}} \frac{d r}{1-r}=2^{-l} \log \log \frac{1}{\varepsilon}<\varepsilon
$$

when $l$ is large enough. Choosing $\varepsilon$ small enough and $l_{0}=l_{0}(\epsilon)$ large enough, we obtain the desired inequality (6.10).

This completes the proof of Theorem 1.2

6.5. Concluding remark. It is interesting to compare the behavior of the function defined by (6.1) with the results on radial growth obtained in [6]. Calculations of the last section show that there exists a set $F \subset[0,1]$ such that

and

$$
|F \cap[r, 1]|=O\left((1-r)^{2} \log \left(\frac{1}{1-r}\right)^{c}\right) \quad(r \rightarrow 1)
$$

$$
\liminf _{r \nearrow 1, r \notin F} \frac{u\left(r e^{i \phi}\right)}{\log \frac{1}{1-r}}>0
$$

for almost each $\phi \in(-\pi, \pi]$. Thus function $u$ growth as $\log \frac{1}{1-r}$ along almost every radius when we delete a system of very thin rings from the unit disc.

\section{REFERENCES}

[1] J.M. Anderson, L.D. Pitt, Probabilistic behaviour of functions in the Zygmund classes $\Lambda^{*}$ and $\lambda^{*}$, Proc. London Math. Soc., (3) 59 (1989), no. 3, 558-592.

[2] R. Bass, Probabilistic techniques in analysis, Springer-Verlag, New York, 1995.

[3] R. Bañuelos, I. Klemes, and C.N. Moore, An analogue for harmonic functions of Kolmogorov's law of the iterated logarithm, Duke Math. J., 57 (1988), no.1, 37-68.

[4] R. Bañuelos, C.N. Moore, Probabilistic behavior of Harmonic functions, Birkhäuser, 1999.

[5] A. Borichev, Yu. Lyubarskii, Uniqueness theorems for Korenblum type spaces, J. Anal. Math., 103 (2007), 307-329.

[6] A. Borichev, Yu. Lyubarskii, E. Malinnikova, P. Thomas, Radial growth of functions in the Korenblum space, Algebra i Analiz, 21 (2009), no. 6. (see also arXiv:0903.5125)

[7] D.L. Burkholder, R.F. Gundy, Extrapolation and interpolation of quasilinear operators on martingales, Acta Math., 124 (1970), 249-304.

[8] C.Y. Chang, J.M. Wilson, and T.H. Wolf, Some weighted norm inequalities concerning the Srödinger operator, Comment. Math. Helv., 60 (1985), 217-246.

[9] K.S. Eikrem, E. Malinnikova, Radial behavior of harmonic functions in the unit ball, Preprint, 2010 
[10] H. Hedenmalm, B. Korenblum, K. Zhu, Theory of Bergman spaces, Graduate Texts in Mathematics, 199. Springer-Verlag, New York, 2000.

[11] J.-P. Kahane, Y. Katznelson, Sur le comportement radial des fonctions analytiques. (French) C. R. Acad. Sci. Paris Ser. A-B 272 (1971) A718-A719.

[12] J.-P. Kahane, M. Weiss, and G. Weiss, On lacunary power series, Arkiv for Matematik, 5 (1963), no. 1-2, 1-26.

[13] B. Korenblum, An extension of the Nevanlinna theory, Acta Math. 135 (1975), no. 3-4, 187-219.

[14] N. Makarov, On the distortion of boundary sets under conformal mappings. Proc. London Math. Soc., 351 (1985), no. 2, 369-384.

[15] X. Massaneda, P. Thomas, Phragmén-Lindelöf-type problems for $A^{-\alpha}$. Bergman spaces and related topics in complex analysis, 153-163, Contemp. Math., 404, Amer. Math. Soc., Providence, RI, 2006.

[16] I. I. Privalov, Boundary properties of analytic functions (Russian), Gosudarstv. Izdat. Tehn.-Teor. Lit., Moscow-Leningrad, 1950.

[17] K. Seip, Interpolation and sampling in spaces of analytic functions University Lecture Series, 33. American Mathematical Society, Providence, RI, 2004.

[18] W.F. Stout, A martingale analogue of Kolmogorov's law of the iterated logarithm, Z. Wahrscheinlichkeitstheorie und Verw. Gebiete, 15 (1970), 279-290.

[19] W.F. Stout, Almost sure convergence. Probability and Mathematical Statistics, Vol. 24. Academic Press, New York-London, 1974.

[20] M. Weiss, The law of the iterated logarithm for lacunary trigonometric series, Trans. AMS, 91 (1959), no. 3, 444-469.

Department of Mathematical Sciences, Norwegian University of Science and Technology, NO-7491, Trondheim, Norway

E-mail address: yura@math.ntnu.no

Department of Mathematical Sciences, Norwegian University of Science And Technology, NO-7491, Trondheim, Norway

E-mail address: eugenia@math.ntnu.no 